\title{
Construcción de una cartera de inversión usando modelos GARCH
}

\begin{abstract}
RESUMEN
En este artículo se analizan portafolios de inversiones con títulos accionarios del mercado chileno, usando el modelo de media varianza propuesto por Markowitz, particularmente, mediante la utilización de la matriz de varianza-covarianza no condicional y condicional, donde esta última es estimada a través de modelos GARCH. Posteriormente, se evalúa el desempeño de estas carteras usando como referente (benchmark) un portafolio de mercado representado por el índice general de precio de acciones (IGPA) de la bolsa de Comercio de Santiago de Chile.
\end{abstract}

Palabras claves: portafolios, GARCH, evaluación.

\section{BUILDING AN INVESTMENT PORTFOLIO USING GARCH \\ ABSTRACT}

In this paper we analyze investment portfolios of Chilean market, using the average model variance proposed by Markowitz, particularly using the variance-covariance matrix of unconditional and conditional, where the latter is estimated through GARCH models. Then, we evaluate the performance of these portfolios using as a reference (benchmark) a market portfolio given by the general index of share prices (IGPA) of Chilean market.

Keywords: portfolios, GARCH, evaluation.

\section{INTRODUCCIÓN}

Los mercados accionarios representan un enorme desafío para los inversionistas, ya que la dinámica que presentan los precios en estos mercados está gobernada por múltiples factores que hacen que el valor de las acciones esté sujeto a muchas fluctuaciones Fama (1963), impidiendo la construcción de modelos de predicción adecuados que sean capaces de entregar proyecciones que no tengan un margen de error importante.

Uno de los problemas que surgen precisamente por la dinámica que tienen los mercados financieros, es la forma en que se puede construir una cartera o portafolio de inversión, tomando en cuenta variables como el riesgo y las rentabilidades asociadas a cada componente de la cartera (acciones en este caso) para obtener una máxima rentabilidad con un mínimo de riesgo.

En este trabajo, se elaborarán modelos para construir carteras de inversión, desde el punto de vista de la teoría de cartera de Markowitz, usando modelos GARCH Nelson (1991) para la estimación de las volatilidades de los activos que conformarán los portafolios, comparando su desempeño con carteras formadas con el mismo modelo, pero que no usan los GARCH en la estimación de la volatilidad Fernández (2006).

\section{ANÁLISIS TEÓRICO}

\section{El modelo de Markowitz}

El enfoque propuesto por Harry Markowitz en la década de los cincuenta sobre la conformación de portafolios de inversión, revolucionó el campo de las finanzas, entregando principios, como el de portafolios eficientes, que están presentes en una gran cantidad de modelos de construcción de carteras, conservando de esta forma la esencia de la propuesta seminal de Markowitz.

1 Doctor en Finanzas, profesor del Departamento de Economía Y Finanzas de la Universidad del Bío Bío, Concepción, Chile. E-mail: mauroguti40@yahoo.es

2 Ingeniero en estadística de la Universidad del Bío Bío, Concepción, Chile.

E-mail: m salgadoib@yahoo.es 
Un portafolio eficiente, según Markowitz, es aquel que tiene un mínimo riesgo para un retorno dado o, equivalentemente un portafolio con un máximo retorno para un nivel de riesgo dado Markowicz (1959).

Una de las formas de encontrar este conjunto de portafolios eficientes es a través del siguiente modelo, que solo considera la minimización de la varianza del portafolio y que corresponde al siguiente esquema de programación no lineal.

$$
\begin{array}{ll}
\text { Min } & \sigma_{c}^{2} \\
s / a & \\
& \sum_{i=1}^{N} \bar{R}_{i} x_{i}=R \\
& \sum_{i=1}^{N} x_{i}=1
\end{array}
$$

En este problema de optimización se pueden distinguir los siguientes elementos.

\section{Función objetivo}

$$
\sigma_{c}^{2}=\sum_{i=1}^{N} \sum_{j=1}^{N} x_{i} x_{j} \sigma_{i j}
$$

\section{Restricciones}

Las restricciones están representadas por:

- $R$, que es el valor esperado de los retornos del portafolio y que se fijan en un valor $R$.

$$
\sum_{i=1}^{N} x_{i}=1
$$

, esto corresponde a que la suma de

- cada proporción o ponderación de cada activo, que compondrá al portafolio o cartera de inversión debe sumar uno.

Una vez que el problema es resuelto con alguna técnica de programación matemática, se logra obtener la proporción de cada activo dentro de la cartera de inversiones, y que satisfacen las restricciones ${ }^{3}$ planteadas en el modelo.

La solución de este modelo, en un principio dió una gran cantidad de problemas ya que al aumentar el número de activos en la cartera, también aumentó la cantidad de operaciones en la determinación de las covarianzas entre los activos.
Esto dio origen al modelo diagonal de Sharpe (1970), el cual simplificó el modelo de Markowitz, y de paso entregó una expresión que permitía relacionar los retornos accionarios con los movimientos del mercado, a través de un índice representativo de este.

\section{El portafolio óptimo de mínima varianza}

La varianza mínima global es la varianza más pequeña posible que se puede encontrar para un portafolio dado, la cual lleva asociada una rentabilidad esperada dada para dicho portafolio, y por tanto, si el inversionista quisiera aumentar el retorno esperado de su portafolio, tendría que asumir un mayor valor para dicha varianza $o$, en otras palabras, vería incrementado su nivel de riesgo a la par con el aumento de su rentabilidad esperada (Glosten et al 1993).

Para determinar el mínimo global en el problema de determinación de una cartera de inversiones óptima, se procede a resolver un problema de optimización no lineal en el cual se usa una técnica conocida como el método de los multiplicadores de Lagrange que nos permitirá encontrar la proporción en la que se encuentra cada activo dentro de la cartera y que minimizan la varianza de la misma Carol (2001).

El problema a resolver es:

$$
\begin{gathered}
\text { Min } \sigma_{c}^{2}=\sum_{i=1}^{N} \sum_{j=1}^{N} x_{i} x_{j} \sigma_{i j} \\
S / a \\
\sum_{1=1}^{N} x_{i}=1
\end{gathered}
$$

Usando multiplicadores de Lagrange, tenemos la siguiente función a minimizar:

$$
Z=\sigma_{c}^{2}+\lambda\left(1-x_{1}-x_{2}-x_{3}-\ldots-x_{N}\right)
$$

Para encontrar los extremos de esta función se debe resolver el siguiente sistema de ecuaciones. 


$$
\begin{aligned}
& \frac{\partial Z}{\partial x_{1}}=2 \sigma_{1} x_{1}+2 \sigma_{12} x_{2}+2 \sigma_{1 a} x_{1}+\ldots+2 \sigma_{1 N} x_{N}-\lambda=0 \ldots \\
& \frac{\partial Z}{\partial x_{2}}=2 \sigma_{21} x_{1}+2 \sigma_{2} x_{2}+2 \sigma_{2 a} x_{3}+\ldots .+2 \sigma_{2 H} x_{N}-\lambda=0 \ldots
\end{aligned}
$$

$$
\begin{aligned}
& \frac{\partial Z}{\partial x_{H}}=2 \sigma_{N} x_{1}+2 \sigma_{N} x_{2}+2 \sigma_{N} x_{1}+\ldots+2 \sigma_{H} x_{N}-\lambda=0 \\
& \frac{\partial Z}{\partial z}=1-x_{1}-x_{2}-x_{1}-\ldots-x_{H}=0 \quad \ldots \ldots(N+1)
\end{aligned}
$$

Tomando las $\mathrm{N}$ primeras ecuaciones, se tiene el siguiente sistema de ecuaciones:

$$
\begin{aligned}
& \frac{\partial Z}{\partial x_{1}}-2 \sigma_{1} x_{1}+2 \sigma_{12} x_{2}+2 \sigma_{13} x_{3}+\ldots+2 \sigma_{1 N} x_{N}-\lambda-0 \ldots \\
& \frac{\partial Z}{\partial x_{2}}-2 \sigma_{21} x_{1}+2 \sigma_{2} x_{2}+2 \sigma_{20} x_{3}+\ldots+2 \sigma_{2 N} x_{H}-\lambda-0 \ldots \\
& \frac{\partial Z}{\partial x_{N}}-2 \sigma_{N} x_{1}+2 \sigma_{H} x_{2}+2 \sigma_{H} x_{3}+\ldots+2 \sigma_{H N} x_{N}-\lambda-0 \ldots
\end{aligned}
$$

Escribiendo dicho sistema en forma matricial se tendrá:

$$
\underbrace{\left[\begin{array}{ccccc}
\sigma_{1}^{2} & \sigma_{12} & \sigma_{13} & \ldots & \sigma_{1 N} \\
\sigma_{21} & \sigma_{2}^{2} & \sigma_{23} & \ldots & \sigma_{2 N} \\
\cdot & \cdot & \cdot & \cdot & \cdot \\
\cdot & \cdot & \cdot & \cdot & \cdot \\
\sigma_{N 1} & \sigma_{N 2} & \sigma_{N 3} & \cdots & \sigma_{N}^{2}
\end{array}\right]}_{\mathbf{A}} \underbrace{\left[\begin{array}{c}
x_{1} \\
x_{2} \\
\cdot \\
x_{N}
\end{array}\right]}_{\mathbf{W}}=\frac{1}{2} \underbrace{\left[\begin{array}{c}
\lambda \\
\lambda \\
\cdot \\
\lambda
\end{array}\right]}_{\tilde{\mathbf{e}}}
$$

Luego, queda la siguiente ecuación matricial:

$$
\text { Aw }=\frac{1}{2}
$$

Considerando que $\ddot{\mathrm{e}}=\lambda \mathbf{u}$, donde $\mathbf{u}=\left[\begin{array}{c}1 \\ 1 \\ \cdot \\ \cdot \\ 1\end{array}\right]$, la expresión (1) se escribirá como: $A w=\frac{1}{2} \lambda u$
Resolviendo para $\mathbf{w}$ en (1) se tiene:

$$
\mathbf{w}=\mathbf{A}^{-1} \frac{1}{2} \lambda \mathbf{u}
$$

Tomando la ecuación $\mathrm{N}+1$ del sistema original y escribiéndola en forma matricial se tendrá:

$$
\mathbf{u}^{\mathrm{t}} \mathbf{w}=1
$$

Debemos encontrar el valor de $\lambda$ para conocer el valor de los componentes del vector columna $\mathbf{w}$. Para lograr esto, sustituimos la expresión (2) en (3) encontrándose una expresión para $\lambda$ de la forma:

$$
\text { forma: } \lambda=\frac{2}{\sum_{i=1}^{N} \sum_{j=1}^{N} a_{i j}} \text { (4) }
$$

Ahora, sustituyendo la expresión dada por (4) en (2), quedará:

$$
\mathbf{w}=\frac{1}{\sum_{i=1}^{N} \sum_{j=1}^{N} a_{i j}} \mathbf{A}^{-1} \mathbf{u}
$$


Haciendo, $\quad \mathbf{A}^{-1} \mathbf{u}=\left[\begin{array}{c}\sum_{j=1}^{N} a_{1 j} \\ \sum_{j=1}^{N} a_{2 j} \\ \cdot \\ \cdot \\ \sum_{j=1}^{N} a_{N j}\end{array}\right]$ y reemplazando en (5)

finalmente se tendrá $\mathbf{w}=\left[\begin{array}{c}\frac{\sum_{j=1}^{N} a_{1 j}}{\sum_{i=1}^{N} \sum_{j=1}^{N} a_{i j}} \\ \frac{\sum_{j=1}^{N} a_{2 j}}{\sum_{i=1}^{N} \sum_{j=1}^{N} a_{i j}} \\ \cdot \\ \cdot \\ \frac{\sum_{j=1}^{N} a_{N j}}{\sum_{i=1}^{N} \sum_{j=1}^{N} a_{i j}}\end{array}\right]$

El vector columna dado en la expresión (6) representa la solución al problema de optimización. En él están contenidos los pesos o ponderaciones para cada uno de los activos financieros que compondrán la cartera de inversión y que cumplen con la condición de minimizar la varianza de la cartera. Así, por ejemplo, tendremos que la ponderación del i-ésimo activo dentro del portafolio será:

$$
x_{i}=\frac{\sum_{j=1}^{N} a_{i j}}{\sum_{i=1}^{N} \sum_{j=1}^{N} a_{i j}}
$$

La solución analítica planteada hasta aquí se puede resumir en una serie de pasos que permiten implementar esta solución y que son los siguientes:

1. Determinar la matriz de varianza-covarianza para los retornos de los activos que compondrán la cartera.

2. Determinar la matriz inversa de la matriz de varianza covarianza.

3. Sumar cada fila de la matriz inversa de la matriz

de varianza-covarianza, es decir $\sum_{j=1}^{N} a_{i j}$, desde $i=1, \ldots, N$.
4. Obtener la suma de todos los elementos que conforman la matriz inversa, es decir, $\sum_{i=1}^{N} \sum_{j=1}^{N} a_{i j}$.

5. Calcular la proporción dada por la expresión (7) para cada uno de los activos, es decir, determinar $x_{i}$ desde $i=1, \ldots, N$.

En conclusión, se tiene que la varianza o desviación mínima para la cartera será:

$$
\sigma_{\min c}=\sqrt{\mathbf{w}^{\mathrm{t}} \mathbf{A w}}
$$

A la cual corresponde un retorno esperado de:

$$
R_{\min c}=\mathbf{w}^{\mathrm{t}} \overline{\mathbf{R}}
$$

Estos valores determinan un punto cuyas coorde-

nadas son $\left(\sigma_{\min c}, R_{\min c}\right)$ en el plano de retornoriesgo $^{2}$ y que constituye el punto de referencia, a partir del cual se construye la frontera eficiente.

\section{La frontera eficiente}

La frontera eficiente es el conjunto de puntos del plano de retorno-riesgo, en la cual están todas las carteras de inversión que tienen un mínimo riesgo para un retorno esperado dado Sharpe (1970).

Cuando se usa optimización para obtener la frontera eficiente se debe resolver el problema de optimización para cada nivel de retorno $R$ elegido por el inversionista.

En el presente trabajo se usa esta misma lógica para resolver dicho problema, pero se describe un método que puede resultar más cómodo y que está basado en los multiplicadores de Lagrange.

Sea el siguiente problema de optimización para un nivel de retorno $\mathrm{R}$ dado.

$$
\begin{aligned}
\operatorname{Min} \sigma_{p}^{2} & =\sum_{i=1}^{N} \sum_{j=1}^{N} x_{i} x_{j} \sigma_{i j} \\
S / a & \\
\sum_{1=1}^{N} x_{i} & =1 \\
\sum_{1=1}^{N} \bar{R}_{i} x_{i} & =R
\end{aligned}
$$

$2 \quad$ El plano de retorno-riesgo está formado por todos los puntos cuyas coordenadas son de la forma $\left(\sigma_{c}, R_{c}\right)$. 
La función a minimizar es:

$$
Z=\sigma_{c}^{2}+\lambda_{1}\left(1-x_{1}-x_{2}-x_{3}-\ldots-x_{N}\right)+\lambda_{2}\left(R-R_{1} x_{1}-R_{2} x_{2}-R_{3} x_{3}-\ldots-R_{N} x_{N}\right)
$$

Para hallar el valor mínimo de esta expresión, se debe resolver el siguiente sistema de ecuaciones:

$$
\begin{aligned}
& \frac{\partial Z}{\partial x_{1}}=2 \sigma_{1}^{2} x_{1}+2 \sigma_{12} x_{2}+2 \sigma_{13} x_{3}+\ldots .+2 \sigma_{1 N} x_{N}-\lambda_{1}-\lambda_{2} R_{1}=0 \quad \ldots \\
& \frac{\partial Z}{\partial x_{2}}=2 \sigma_{21} x_{1}+2 \sigma_{2}^{2} x_{2}+2 \sigma_{23} x_{3}+\ldots .+2 \sigma_{2 N} x_{N}-\lambda_{1}-\lambda_{2} R_{2}=0 \ldots \\
& \cdot \\
& \cdot \\
& \frac{\partial Z}{\partial x_{N}}=2 \sigma_{N 1} x_{1}+2 \sigma_{N 2} x_{2}+2 \sigma_{N 3} x_{3}+\ldots .+2 \sigma_{N N}^{2} x_{N}-\lambda_{1}-\lambda_{2} R_{N}=0 \ldots \\
& \frac{\partial Z}{\partial \lambda_{1}}=1-x_{1}-x_{2}-x_{3}-\ldots-x_{N}=0 \quad \ldots . . .(N+1) \\
& \frac{\partial Z}{\partial \lambda_{2}}=R-R_{1} x_{1}-R_{2} x_{2}-R_{3} x_{3}-\ldots-R_{N} x_{N}=0 \ldots(N+2)
\end{aligned}
$$

Considerando las $\mathrm{N}$ primeras ecuaciones nos queda el sistema siguiente.

$$
\begin{aligned}
& \frac{\partial Z}{\partial x_{1}}=2 \sigma_{1}^{2} x_{1}+2 \sigma_{12} x_{2}+2 \sigma_{13} x_{3}+\ldots .+2 \sigma_{1 N} x_{N}-\lambda_{1}-\lambda_{2} R_{1}=0 \ldots \\
& \frac{\partial Z}{\partial x_{2}}=2 \sigma_{21} x_{1}+2 \sigma_{2}^{2} x_{2}+2 \sigma_{23} x_{3}+\ldots .+2 \sigma_{2 N} x_{N}-\lambda_{1}-\lambda_{2} R_{2}=0 \ldots \\
& \cdot \\
& \frac{\partial Z}{\partial x_{N}}=2 \sigma_{N 1} x_{1}+2 \sigma_{N 2} x_{2}+2 \sigma_{N 3} x_{3}+\ldots .+2 \sigma_{N N}^{2} x_{N}-\lambda_{1}-\lambda_{2} R_{N}=0 \ldots
\end{aligned}
$$

Al escribir este último sistema en forma matricial se tendrá:

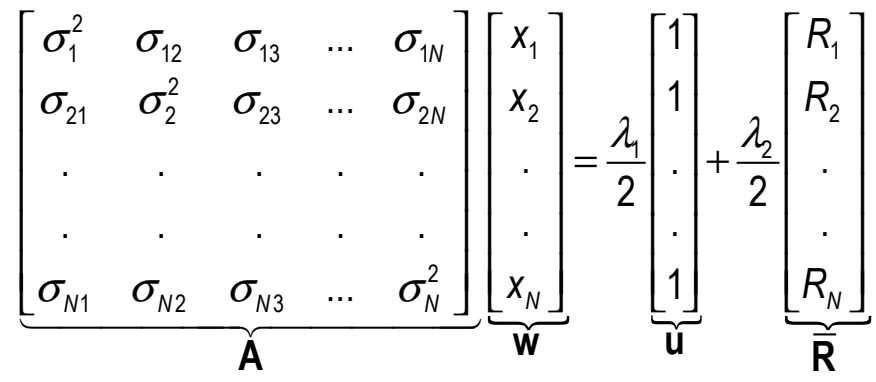


Luego, la ecuación matricial será:

$$
\mathrm{Aw}=\frac{\lambda_{1}}{2} \mathbf{u}+\frac{\lambda_{2}}{2} \overline{\mathbf{R}}
$$

Resolviendo (1) para w queda:

$$
\mathbf{w}=\frac{\lambda_{1}}{2} A^{-1} u+\frac{\lambda_{2}}{2} A^{-1} \bar{R}
$$

Escribiendo en términos matriciales las ecuaciones $\mathrm{N}+1$ y N+2, se tendrá:

$$
\begin{aligned}
\mathbf{u}^{\mathbf{t}} \mathbf{w} & =1 \\
\overline{\mathbf{R}}^{\mathbf{t}} \mathbf{w} & =R
\end{aligned}
$$

Al sustituir el valor de $\mathbf{w}$, obtenido en (2) en las expresiones (3) y (4) respectivamente, se obtiene el siguiente sistema de ecuaciones:

$$
\begin{gathered}
\lambda_{1} \mathbf{u}^{\mathbf{t}} \mathbf{A}^{-1} \mathbf{u}+\lambda_{2} \mathbf{u}^{\mathbf{t}} \mathbf{A}^{-1} \overline{\mathbf{R}}=2 \\
\lambda_{1} \overline{\mathbf{R}}^{\mathbf{t}} \mathbf{A}^{-1} \mathbf{u}+\lambda_{2} \overline{\mathbf{R}}^{\mathbf{t}} \mathbf{A}^{-1} \overline{\mathbf{R}}=2 R
\end{gathered}
$$

Haciendo $\gamma_{1}=\mathbf{u}^{\mathbf{t}} \mathbf{A}^{-1} \mathbf{u}, \gamma_{2}=\mathbf{u}^{\mathbf{t}} \mathbf{A}^{-1} \overline{\mathbf{R}}, \gamma_{3}=\overline{\mathbf{R}}^{\mathrm{t}} \mathbf{A}^{-1} \mathbf{u}$ y $\gamma_{4}=\overline{\mathbf{R}}^{\mathrm{t}} \mathbf{A}^{-1} \overline{\mathbf{R}}$ y, al reemplazar estás expresiones en el sistema anterior se tiene:

$$
\begin{gathered}
\lambda_{1} \gamma_{1}+\lambda_{2} \gamma_{2}=2 \\
\lambda_{1} \gamma_{3}+\lambda_{2} \gamma_{4}=2 R
\end{gathered}
$$

Resolviendo dicho sistema se obtienen los valores para las incógnitas $\lambda_{1}$ y $\lambda_{2}$ :

$$
\begin{gathered}
\lambda_{2}=\frac{2\left[R \gamma_{1}-\gamma_{3}\right]}{\gamma_{4} \gamma_{1}-\gamma_{2} \gamma_{3}} \\
\lambda_{1}=\frac{2-\lambda_{2} \gamma_{2}}{\gamma_{1}}
\end{gathered}
$$

Luego, las ecuaciones de trabajo son:

$$
\begin{aligned}
& \mathbf{w}=\frac{\lambda_{1}}{2} \mathbf{A}^{-1} \mathbf{u}+\frac{\lambda_{2}}{2} \mathbf{A}^{-1} \mathbf{R} \\
& \lambda_{1}=\frac{2-\lambda_{2} \gamma_{2}}{\gamma_{1}} \\
& \lambda_{2}=\frac{2\left[R \gamma_{1}-\gamma_{3}\right]}{\gamma_{4} \gamma_{1}-\gamma_{2} \gamma_{3}}
\end{aligned}
$$

Para poder utilizar estas ecuaciones es necesario determinar los valores para $\gamma_{1}, \gamma_{2}, \gamma_{3}$ y $\gamma_{4}$, los cuales se obtienen a través de operaciones matriciales entre la inversa $\mathrm{A}^{-1}$ de la matiz de varianza, covarianza y los vectores columna u y $\overline{\mathbf{R}}$.

Una vez que estos valores son determinados se procede a reemplazarlos en las ecuaciones de trabajo.

Estas ecuaciones permiten encontrar puntos sobre la frontera eficiente, que estarán dados por el par $\left(\sigma_{c}, R_{c}\right)$, en donde $R_{c}>R_{c \min }$. Es decir, para cada valor dado de $R_{c}$ se obtendrá el correspondiente valor para $\sigma_{c}$, la que será determinada a través de:

$$
\sigma_{c}=\sqrt{w^{t} A w}
$$

El vector $\mathbf{w}$ de ponderadores así obtenido, permiten la obtención de un conjunto de carteras eficientes de las cuales podrá disponer el inversionista.

Como se ha visto hasta aquí, la determinación de carteras óptimas a través del modelo de media varianza de Markowitz, requiere como principal insumo las series de retornos de los activos que conformarán el portafolio de inversión. Es por esto, que a continuación se explicarán algunas de las principales propiedades de las series de retornos financieros y de cómo estas pueden ser analizadas.

\section{Tipos de modelos GARCHs}

Existe una serie de variantes de estos modelos que principalmente se diferencian entre sí, esencialmente por la forma en que consideran el grado de asimetría que presentan los retornos en la serie Fernández (2006), y de cómo estas asimetrías influyen en los valores de la varianza observada.

Es muy conocido el hecho de que en muchas series financieras, los valores negativos en los retornos tienen un mayor impacto en la volatilidad que valores positivos, lo cual se conoce como apalancamiento.

También, en algunas ocasiones, los retornos incluyen una prima al riesgo, lo que origina a un tipo particular de modelos (Carol 2001).

Algunas de las variantes más conocidas son:

IGARCH O GARCH integrado, presentado por Engle y Bollerslev en el año 1986.

$$
\sigma_{t}^{2}=\alpha \varepsilon_{t-1}^{2}+(1-\alpha) \sigma_{t-1}^{2}
$$


Este modelo considera la persistencia en la varianza condicional heterocedástica.

TS-GARCH de Schwert propuesto en 1989, permite corregir efectos de asimetría en las variaciones al alza y a la baja.

$$
\sigma_{t}=\alpha_{0}+\alpha_{1} \sigma_{t-1}+\alpha_{2} \sigma_{t-1}\left|\varepsilon_{t-1}^{2}\right|
$$

E-GARCH o GARCH exponencial formulado por Nelson en 1991 para incluir efectos de asimetría en los datos, cuya expresión es:

$$
\ln \sigma_{t}^{2}=\omega+g\left(z_{t-1}\right)+\beta \ln \sigma_{t-1}^{2}
$$

En donde, $g\left(z_{t}\right)=\lambda z_{t}+\varphi\left(\left|z_{t}\right|-\sqrt{2 / \pi}\right)$ es conocida como función de respuesta asimétrica y la variable $\mathrm{z}_{\mathrm{t}}$ es una normal estándar. Cuando $\varphi>0$, y $\lambda<0$, los impactos negativos en los retornos tienen un mayor valor en la varianza que impactos positivos.

GJR-GARCH o GJR formulado por Glosten, Jagannanthan y Runkle en el año 1993, sugiere que la varianza condicional presenta una respuesta diferente a las innovaciones pasadas negativas y positivas (efecto leverage).

El modelo $\operatorname{GJR}(1,1)$ se expresa como:

$$
\sigma_{t}^{2}=\omega+\alpha \varepsilon_{t-1}^{2}+\gamma \varepsilon_{t-1}^{2} I\left(\varepsilon_{t-1}<0\right)+\beta \sigma_{t-1}^{2}
$$

Donde $I(\cdot)$ es la función indicadora. En este modelo la volatilidad es proporcionalmente más grande para innovaciones pasadas negativas que positivas.

También, las propuestas de estos modelos se han modificado en su generalización a esquemas multivariados, pero presentan el inconveniente de tener que estimar una gran cantidad de parámetros en la medida que aumenta el número de series de retornos a analizar. Entre los modelos más conocidos se encuentran:

MGARCH o GARCH multivariado propuesto por Bollerslev, Engle y Wooldridge en 1988.

La formulación general para un MGARCH $(p, q)$ es :

$\operatorname{vech}\left(\Omega_{t}\right)=\Omega+\sum_{i=1}^{q} A_{i} \operatorname{vech}\left(\varepsilon_{t-i} \varepsilon_{t-i}^{\prime}\right)+\sum_{i=1}^{p} B_{i} \operatorname{vech}\left(\Omega_{t-i}\right)$

Donde vech(.) es un operador que permite descomponer la matriz $\Omega_{t}$ de covarianza condicional. Este modelo es muy complejo, ya que no se garantiza que la matriz de covarianza condicional estimada sea definida positiva; por otra parte, su implementación computacional no está exenta de dificultades ya que la cantidad de parámetros que se deben estimar es enorme incrementándose los costos en este sentido.

Se han realizado esfuerzos para desarrollar formas más parsimoniosas, en el esquema multivariado y que también garanticen que la matriz de covarianza condicional estimada sea definida positiva, surgiendo interesantes formulaciones auxiliadas por modelos de tipo financiero y que contemplan el uso de modelos GARCH univariados para determinar la matriz de covarianza condicional.

\section{Propiedades de los modelos GARCHs}

Para ilustrar algunas de las propiedades que poseen los modelos de heterocedasticidad condicional autorregresiva, se considerará el caso más simple posible, es decir, el GARCH $(1,1)$. Dichas propiedades permiten afirmar que los modelos GARCHs son capaces de reproducir algunos de los hechos estilizados observados en las series de retornos financieros Engle y Bollerslev (1986).

Sea

$$
\begin{aligned}
a_{t}= & \sigma_{t} \varepsilon_{t} \\
& \sigma_{t}^{2}=\alpha_{0}+\alpha_{1} a_{t-1}^{2}+\beta_{1} \sigma_{t-1}^{2}
\end{aligned}
$$

Con $\alpha_{0}>0,\left(\alpha_{1}, \beta_{1}\right) \geq 0$ y $\varepsilon_{t} \sim N(0,1)$

\section{a. La media y la varianza condicional no son constantes a través del tiempo.}

Para el caso de la media condicional se tiene:

$$
E\left(y_{t} \mid Y_{t-1}\right)=E\left(\sigma_{t} \varepsilon_{t} \mid Y_{t-1}\right)=\sigma_{t} \cdot 0=0
$$

$Y$ para la varianza condicional será:

$$
\operatorname{Var}\left(y_{t} \mid Y_{t-1}\right)=E\left[\left(\sigma_{t} \varepsilon_{t}\right)^{2} \mid Y_{t-1}\right]=\sigma_{t}^{2} E\left[\varepsilon_{t}^{2} \mid Y_{t-1}\right]=\sigma_{t}^{2} \cdot 1=\sigma_{t}^{2}
$$

Por lo tanto, la distribución condicional del proceso será:

$$
y_{t} \mid Y_{t-1} \sim N\left(0, \sigma_{t}^{2}\right)
$$

\section{b. La media y la varianza incondicionales son constantes a través del tiempo.}

Para la media, se tiene:

$$
E\left[y_{t}\right]=E\left(E\left(y_{t} \mid Y_{t-1}\right)\right)=E(0)=0
$$


En el caso de la varianza:

$$
\begin{aligned}
\operatorname{Var}\left(y_{t}\right) & =E\left(\operatorname{Var}\left(y_{t} \mid Y_{t-1}\right)\right)+\operatorname{Var}\left(E\left(y_{t} \mid Y_{t-1}\right)\right) \\
& =E\left(\sigma_{t}^{2}\right)+\operatorname{Var}(0) \\
& =\alpha_{0}+\alpha_{1} \operatorname{Var}\left(y_{t}\right)+\beta_{1} \operatorname{Var}\left(y_{t}\right) \\
& =\frac{\alpha_{0}}{\left(1-\left(\alpha_{1}+\beta_{1}\right)\right)}
\end{aligned}
$$

Como $\alpha_{1}+\beta_{1}<1$, la varianza es constante.

En consecuencia, la serie es estacionaria de segundo orden.

\section{c. No normalidad de la serie.}

Sea:

Con lo que se demuestra claramente que el proceso GARCH es capaz de presentar observaciones no normales que presentan exceso de kurtosis.

$$
\kappa=\frac{E\left(y_{t}^{4}\right)}{\left[\operatorname{Var}\left(y_{t}\right)\right]^{2}}=\frac{3\left[1-\left(\alpha_{1}-\beta_{1}\right)^{2}\right]}{\left[1-\left(\alpha_{1}-\beta_{1}\right)^{2}-2 \alpha_{1}^{2}\right]}>3
$$

\section{d. Autocorrelación nula.}

Para un proceso GARCH estará definida de la siguiente manera:

$$
\rho(k)=\frac{\left[E\left(y_{t} y_{t-k}\right)-E\left(y_{t}\right) E\left(y_{t-k}\right)\right]}{\operatorname{Var}\left(y_{t}\right)}=\frac{E\left(y_{t} y_{t-k}\right)}{\operatorname{Var}\left(y_{t}\right)}
$$

Pero, se tiene $E\left(y_{t} y_{t-k}\right)=E\left[E\left(y_{t} y_{t-k} \mid Y_{t-1}\right)\right]=0$, que al sustituir en (1) se obtiene que:

$$
\rho(k)=0
$$

\section{e. Autocorrelación no nula del cuadrado de las observaciones generadas por el proceso.}

En este caso se debe encontrar la función da autocorrelación para $\mathrm{y}_{\mathrm{t}}^{2}$. Luego, haciendo $u_{t}=y_{t}^{2}-\sigma_{t}^{2}$ y sustituyendo este valor en la expresión para $\sigma_{t}^{2}$, se tendrá:

$$
u_{t}=y_{t}^{2}-\left(\alpha_{0}+\alpha_{1} y_{t-1}^{2}+\beta_{1} \sigma_{t-1}^{2}\right)
$$

Ahora, considerando que $\sigma_{t}^{2}=y_{t}^{2}-u_{t}$, se obtiene:

$$
y_{t}^{2}=\alpha_{0}+\left(\alpha_{1}+\beta_{1}\right) y_{t-1}^{2}-\beta_{1} u_{t-1}+u_{t}
$$

Expresión que corresponde a un proceso ARMA $(1,1)$. En consecuencia, se tiene que el cuadrado de los retornos generados a través del proceso GARCH, sigue un proceso de tipo $\mathrm{ARMA}^{3}$ cuya función de autocorrelación muestra autocorrelaciones importantes entre las observaciones.

\section{APLICACIÓN DEL MODELO GARCH}

\section{Muestra}

Los datos que se utilizaron para este trabajo corresponden a títulos accionarios que fueron transados en la Bolsa de Comercio de Santiago de Chile en el período que va desde el 2 de enero de 1995 al 29 de diciembre de 2000.

Existen numerosos criterios que recomiendan los expertos y que permiten elegir los activos (o pasivos) que conformarán un portafolio de inversiones como los que están basados en la liquidez del titulo, la mayor presencia bursátil del mismo, su patrimonio bursátil, etc.

Sin embargo, para los objetivos que se propone este trabajo solamente se considerarán criterios como: el de elegir sólo títulos accionarios, ya que estos poseen la característica de alta volatilidad que es la deseable para elaborar los modelos, y por otro lado, que los títulos elegidos sean tranzados en la bolsa el mayor número de veces en el período considerado, de tal forma que estos activos sean de alguna manera representativos de los movimientos del mercado Johnson (2000).

En consecuencia, los títulos que cumplen con los criterios antes señalados y que conformarán los portafolios de inversión son: COPEC (sector recursos naturales), CAP (sector industria), CTC-A y ENTEL (sector servicios), COLBUN y ENDESA (sector eléctrico).

Respecto de la elección del período de tiempo para elaborar los modelos, este se dividió en dos partes. La primera corresponde al período que va desde el 2 de enero de 1995 al 30 de diciembre de 1999,

3 Los ARMA son procesos que corresponden a series de tiempo. 
y es el período utilizado para hacer las estimaciones necesarias para la formación de los modelos, el período que va desde el 3 de enero de 2000 al 29 de diciembre del mismo año, en el que se mide el desempeño de las carteras de inversión.

\section{Análisis de los retornos accionarios}

En una primera etapa se realiza el diagnóstico de la presencia de hechos estilizados en cada una de las series de retornos utilizadas. Se procedió ha realizar pruebas estadísticas y gráficas sobre las series de retornos accionarios bajo estudio, con el objeto de poder establecer la presencia de algunos de los hechos estilizados.

Tabla 1. Resumen estadístico para retornos accionarios

\begin{tabular}{|c|c|c|c|c|c|c|}
\hline Acción & Media & Máximo & Mínimo & $\begin{array}{c}\text { Desviación } \\
\text { estándar }\end{array}$ & Asimetría & Curtosis \\
\hline COPEC & 0,00021 & 0,132768 & $-0,065041$ & 0,01950 & 0,60982 & 6,21736 \\
\hline CTC-A & 0,00040 & 0,16352 & $-0,13006$ & 0,02069 & 0,66001 & 10,53830 \\
\hline CAP & $-0,00034$ & 0.108110 & -0.077920 & 0,01856 & 0,28629 & 5,60328 \\
\hline COLBUN & $-0,00028$ & 0,14282 & $-0,11017$ & 0,02100 & 0,45440 & 6,80798 \\
\hline ENDESA & $-0,00005$ & 0,17647 & $-0,13636$ & 0,02004 & 0,44674 & 11,91932 \\
\hline ENTEL & 0,00006 & 0,11628 & $-0,10345$ & 0,02138 & 0,52509 & 6,88184 \\
\hline
\end{tabular}

\begin{tabular}{|c|c|c|c|c|c|c|}
\hline Acción & $\begin{array}{c}\text { Exceso } \\
\text { curtosis }\end{array}$ & Test JB & p-valor & Test ADF & p-valor & $\begin{array}{c}\text { Número } \\
\text { observaciones }\end{array}$ \\
\hline COPEC & 3,21736 & 614,63800 & $3,41 \mathrm{E}-134$ & $-30,76135$ & $1.67 \mathrm{E}-36$ & 1246 \\
\hline CTC-A & 7,53830 & 3040,67900 & $0,00 \mathrm{E}+00$ & $-29,29212$ & $1.34 \mathrm{E}-39$ & 1246 \\
\hline CAP & 2,60328 & 368,86270 & $7,99 \mathrm{E}-81$ & $-27,55497$ & $1.85 \mathrm{E}-42$ & 1246 \\
\hline COLBUN & 3,80798 & 795,70730 & $1,64 \mathrm{E}-173$ & $-31,39364$ & $5.26 \mathrm{E}-35$ & 1246 \\
\hline ENDESA & 8,91932 & 4171,64100 & $0,00 \mathrm{E}+00$ & $-30,34622$ & $1.95 \mathrm{E}-37$ & 1246 \\
\hline ENTEL & 3,88184 & 839,57280 & $4,89 \mathrm{E}-183$ & $-27,75210$ & $3.51 \mathrm{E}-42$ & 1246 \\
\hline
\end{tabular}

Los resultados obtenidos muestran retornos promedios negativos para las acciones de: CAP, COLBUN y ENDESA, lo que indica que los precios de las acciones para estas empresas se encontraban a la baja durante el período considerado. En general, se observan desviaciones estándar bastante grandes en relación al promedio, lo que es indicio de una gran variabilidad y dispersión en los retornos.

La evidencia revela la ausencia de normalidad en las series, lo que es corroborado con la aplicación de la prueba asintótica de Jarque Bera (JB) la que resulta significativa (con p-valor muy próximo a cero) para todas las series, permitiendo rechazar la hipótesis de normalidad en la distribución de los retornos. Las series en estudio son sometidas al test de Dikey-Fuller aumentado, para establecer si hay presencia de raíces unitarias en el proceso que genera los datos. Los resultados del test aplicado a todas las series, aparecen en el estadístico para la prueba (ADF) junto al valor-p, el que resulta ser significativo, rechazándose para todas las series, la hipótesis nula sobre la presencia de raíz unitaria en el proceso, lo que permite concluir que las series de retornos son estacionarias. Se observa que las magnitudes de las funciones de autocorrelación (FAC) como para la función de autocorrelación parcial (FACP), para todas las series de retornos presentan valores despreciables (no mayores que 1) en cada uno de los rezagos. A pesar de que la prueba de Box-Ljung aplicada en cada una de estas funciones arroja un valor bastante significativo para las autocorrelaciones, con un valor-p pequeño, para el estadístico de prueba $(Q)$, en consecuencia, las series de retornos presentan una despreciable dependencia lineal entre los retornos, por el contrario, se observa que las series de retornos financieros analizadas presentan dependencia de tipo no lineal.

Dada la evidencia mostrada por los análisis, es posible concluir que las series de retornos accionarios de: COPEC, CTC-A, CAP, ENDESA, COLBUN y ENTEL presentan los hechos estilizados que son: 
aglomerados de volatilidad, distribución distinta de la normal evidenciada en el exceso de curtosis, dependencia lineal despreciable, dependencia no lineal y estacionaridad.

\section{Metodología de ajuste de los GARCH}

La metodología para ajustar los modelos, consistió en aplicar los criterios de información para seleccionar los modelos que mejor se ajustaran a los datos en estudio, se procedió entonces a elaborar, en cada serie de retornos, un grid para cada combinación $\operatorname{GARCH}(p, q)$, donde $p=0,1$,
$2, \ldots 9 ; q=1,2, \ldots 9$ у $\operatorname{AR}(p), p=1,2, \ldots, 9$, en el que aparece el valor para cada uno de los criterios AIC y BIC, que corresponden solamente a los modelos cuyos parámetros estimados resultaron ser significativos al $5 \%$.

Es así que se eligieron los modelos que minimizan el valor del BIC, ya que este último criterio presenta mejores propiedades

Las siguientes tablas presentan los valores para los criterios AIC y BIC, antes mencionados, obtenidos en el proceso de ajuste de cada uno de los modelos a las series de datos.

Tabla 2: Valores del AIC y BIC para diferente GARCH

\begin{tabular}{llllc}
\hline & AR(p) & GARCH(p, $)$ & AIC & BIC \\
\hline COPEC & AR(1) & GARCH(1,1) & 4,00319 & $\mathbf{4 , 0 1 9 6 6}$ \\
& AR(1) & GARCH(1,3) & 3,99977 & 4,02448 \\
\hline CTC-A & AR(1) & GARCH(1,1) & 3,96547 & $\mathbf{3 , 9 8 1 9 5}$ \\
& AR(1) & GARCH(1,5) & 3,94905 & 3,98199 \\
& AR(1) & GARCH(1,7) & 3,94992 & 3,99110 \\
& AR(1) & GARCH(1,9) & 3,94752 & 3,99694 \\
& AR(1) & GARCH(2,1) & 3,96221 & 3,98280 \\
& AR(1) & GARCH(2,3) & 3,96196 & 3,99079 \\
& AR(1) & GARCH(2,5) & 3,95434 & 3,99140 \\
& AR(1) & GARCH(4,2) & 3,95816 & 3,99110 \\
\hline CAP & AR(1) & GARCH(1,1) & 3,91446 & 3,93093 \\
& AR(1) & GARCH(1,2) & 3,91136 & 3,93195 \\
& AR(1) & GARCH(1,3) & 3,90929 & 3,93400 \\
AR(1) & GARCH(1,5) & 3,90256 & 3,93550 \\
AR(1) & GARCH(1,6) & 3,89759 & 3,93466 \\
AR(1) & GARCH(1,9) & 3,89185 & 3,94126 \\
AR(1) & GARCH(3,3) & 3,87446 & 3,90740 \\
AR(1) & GARCH(3,7) & 3,90602 & 3,95544
\end{tabular}

\begin{tabular}{|c|c|c|c|c|}
\hline & $A R(p)$ & $\mathrm{GARCH}(p, q)$ & $\mathrm{AlC}$ & $\mathrm{BIC}$ \\
\hline COLBUN & $\operatorname{AR}(1)$ & $\mathrm{GARCH}(1,1)$ & 4,22033 & 4,23681 \\
\hline ENDESA & $\begin{array}{l}\operatorname{AR}(1) \\
\operatorname{AR}(1) \\
\operatorname{AR}(1) \\
\operatorname{AR}(1) \\
\operatorname{AR}(1) \\
\operatorname{AR}(1)\end{array}$ & $\begin{array}{l}\text { GARCH(1,1) } \\
\text { GARCH }(1,2) \\
\operatorname{GARCH}(1,3) \\
\operatorname{GARCH}(1,6) \\
\operatorname{GARCH}(2,2) \\
\operatorname{GARCH}(3,3)\end{array}$ & $\begin{array}{l}3,93379 \\
3,93444 \\
3,93288 \\
3,92871 \\
3,93223 \\
3,91972\end{array}$ & $\begin{array}{l}3,95026 \\
3,95503 \\
3,95759 \\
3,96577 \\
3,95694 \\
3,95266\end{array}$ \\
\hline ENTEL & $\begin{array}{l}\operatorname{AR}(1) \\
\operatorname{AR}(1) \\
\operatorname{AR}(1) \\
\operatorname{AR}(1) \\
\operatorname{AR}(1) \\
\operatorname{AR}(1) \\
\operatorname{AR}(1) \\
\operatorname{AR}(1) \\
\operatorname{AR}(1)\end{array}$ & $\begin{array}{l}\text { GARCH(1,1) } \\
\text { GARCH }(1,3) \\
\text { GARCH }(2,1) \\
\text { GARCH }(2,3) \\
\text { GARCH }(2,6) \\
\text { GARCH }(2,7) \\
\text { GARCH }(3,3) \\
\text { GARCH }(6,7) \\
\text { GARCH }(6,9)\end{array}$ & $\begin{array}{l}4,00150 \\
3,99794 \\
4,00071 \\
3,99729 \\
3,99644 \\
3,97929 \\
3,98927 \\
3,98736 \\
3,98389 \\
\end{array}$ & $\begin{array}{l}4,01798 \\
4,02265 \\
4,02130 \\
4,02612 \\
4,03762 \\
4,02459 \\
4,02221 \\
4,04913 \\
4,05389 \\
\end{array}$ \\
\hline
\end{tabular}

En estas tablas aparecen destacados en negrita, los valores más pequeños para el criterio BIC con los correspondientes modelos que son los seleccionados para representar la variabilidad de cada uno de los procesos involucrados en los análisis.

\section{Validación de los modelos estimados}

Como los residuos de los modelos estimados presentaron exceso de curtosis se aplicará un backtest, según Fernández (2006), que consistirá en construir un intervalo de confianza para las series de retornos, y observar el numero de violaciones se producen debidas a movimientos extremos en los retornos debidas a alzas bruscas en los precios.

Para construir el intervalo de confianza mencionado, se construirán series para la media y desviación

estándar condicionales. Luego el intervalo de confianza al $5 \%$ estará dado por la expresión:

$$
\mu_{t \mid t-1} \pm 1,645 \hat{\sigma}_{t \mid t-1}
$$

Por lo tanto, la forma que adoptan los intervalos de confianza superior e inferior para estas series es:

$$
\begin{aligned}
& I s=\hat{\mu}_{t \mid t-1}+1.645 \hat{\sigma}_{t \mid t-1} \\
& l i=\hat{\mu}_{t \mid t-1}-1.645 \hat{\sigma}_{t \mid t-1}
\end{aligned}
$$

Se graficaron las series de retornos para todos los activos, junto con los límites de estos intervalos, y se contabilizó el número de violaciones del limite inferior del intervalo producidas en cada uno de ellos. 
Las desviaciones estándares condicionales obtenidas a través de los modelos GARCH estimados para las series de retornos, se muestran en la tabla siguiente.

Tabla 3: Desviaciones estándares condicionales calculadas con GARCH.

\begin{tabular}{|l|l|l|l|l|l|l|}
\hline & COPEC & CTC-A & CAP & COLBUN & ENDESA & ENTEL \\
\hline Desviación & & & & & & \\
\hline Estándar & 0,15255 & 0,12628 & 0,18516 & 0,19721 & 0,16792 & 0,20824 \\
\hline Condicional & & & & & & \\
\hline
\end{tabular}

Pos otro lado, la matriz de covarianza condicional para los activos y que es obtenida usando el procedimiento de descomposición presentado en secciones anteriores de este trabajo, a través de estas desviaciones estándares condicionales, está dada por:

Tabla 4: Matriz de varianza covarianza condicional

\begin{tabular}{|l|c|c|c|c|c|c|}
\hline & COPEC & CTC-A & CAP & COLBUN & ENDESA & ENTEL \\
\hline COPEC & 0,02327 & 0,00943 & 0,01348 & 0,01043 & 0,01221 & 0,01260 \\
\hline CTC-A & 0,00943 & 0,01595 & 0,00925 & 0,00768 & 0,01059 & 0,00862 \\
\hline CAP & 0,01348 & 0,00925 & 0,03428 & 0,00985 & 0,01189 & 0,01343 \\
\hline COLBUN & 0,01043 & 0,00768 & 0,00985 & 0,03889 & 0,01131 & 0,00985 \\
\hline ENDESA & 0,01221 & 0,01059 & 0,01189 & 0,01131 & 0,02820 & 0,01118 \\
\hline ENTEL & 0,01260 & 0,00862 & 0,01343 & 0,00985 & 0,01118 & 0,04336 \\
\hline
\end{tabular}

La matriz de covarianza no condicional es obtenida usando varianzas y covarianzas no condicionales, usando las estimaciones usuales que fueron comentadas en secciones anteriores de este trabajo, y corresponde a:

Tabla 5: Matriz de varianza covarianza no condicional

\begin{tabular}{|l|l|l|l|l|l|l|}
\hline & COPEC & CTC-A & CAP & COLBUN & ENDESA & ENTEL \\
\hline COPEC & 0,00038 & 0,00020 & 0,00017 & 0,00014 & 0,00019 & 0,00017 \\
\hline CTC-A & 0,00020 & 0,00043 & 0,00015 & 0,00013 & 0,00021 & 0,00014 \\
\hline CAP & 0,00017 & 0,00015 & 0,00034 & 0,00011 & 0,00014 & 0,00014 \\
\hline COLBUN & 0,00014 & 0,00013 & 0,00011 & 0,00044 & 0,00014 & 0,00011 \\
\hline ENDESA & 0,00019 & 0,00021 & 0,00014 & 0,00014 & 0,00040 & 0,00014 \\
\hline ENTEL & 0,00017 & 0,00014 & 0,00014 & 0,00011 & 0,00014 & 0,00046 \\
\hline
\end{tabular}

\section{Construcción de la cartera de inversiones}

La elaboración del modelo de la cartera de media varianza es aplicado a los seis activos seleccionados que corresponden a acciones pertenecientes a: COPEC, CTC-A, CAP, COLBUN, ENDESA Y ENTEL.

La inversión se hace considerando datos de frecuencia diaria para las series correspondientes a los activos mencionados, tomando la información de las series hasta el 29 de diciembre de 2000, fecha en que se construye el portafolio, es decir, se realiza la inversión.

\section{Resultados modelo estimación matriz de cova- rianza condicional}

El modelo considera la siguiente información al 29 de diciembre de 2000 que se muestra en la Tabla 6 y Tabla 7.

Tabla 6: Media de los retornos para cada activo en el período considerado.

\begin{tabular}{|c|c|c|c|c|c|c|}
\hline Acción & COPEC & CTC-A & CAP & COLBUN & ENDESA & ENTEL \\
\hline Media & $0,021 \%$ & $0,040 \%$ & $-0,034 \%$ & $-0,028 \%$ & $-0,005 \%$ & $0,006 \%$ \\
\hline
\end{tabular}

Tabla 7: Matriz varianza covarianza condicional (cifras en \%)

\begin{tabular}{|l|c|c|c|c|c|c|}
\hline & COPEC & CTC-A & CAP & COLBUN & ENDESA & ENTEL \\
\hline COPEC & $2,33 \%$ & $0,94 \%$ & $1,35 \%$ & $1,04 \%$ & $1,22 \%$ & $1,26 \%$ \\
\hline CTC-A & $0,94 \%$ & $1,60 \%$ & $0,93 \%$ & $0,77 \%$ & $1,06 \%$ & $0,86 \%$ \\
\hline CAP & $1,35 \%$ & $0,93 \%$ & $3,43 \%$ & $0,99 \%$ & $1,19 \%$ & $1,34 \%$ \\
\hline COLBUN & $1,04 \%$ & $0,77 \%$ & $0,99 \%$ & $3,89 \%$ & $1,13 \%$ & $0,99 \%$ \\
\hline ENDESA & $1,22 \%$ & $1,06 \%$ & $1,19 \%$ & $1,13 \%$ & $2,82 \%$ & $1,12 \%$ \\
\hline ENTEL & $1,26 \%$ & $0,86 \%$ & $1,34 \%$ & $0,99 \%$ & $1,12 \%$ & $4,34 \%$ \\
\hline
\end{tabular}

En donde, la última tabla representa la matriz de varianza covarianza condicional expresada en porcentaje.

De la tabla se puede observar que la variación (retornos) promedio en los precios, señalan que tres de los activos que conformarán los portafolios, tuvieron una variación promedio negativa debida a una tendencia a la baja en los precios durante el período considerado.

La mayor caída la registra el CAP, con una volatilidad bastante fuerte de un 3,43\%, le sigue COLBUN que también presenta una alta volatilidad la que corresponde a un $3,89 \%$. 
Los activos con tendencias al alza son: COPEC, CTC-A y ENTEL, registrando la mayor tendencia
CTC-A, la que también es la menos volátil con solo un $1,6 \%$.

Gráfico N ${ }^{\circ}$ 1. Frontera eficiente matriz de covarianza condicional

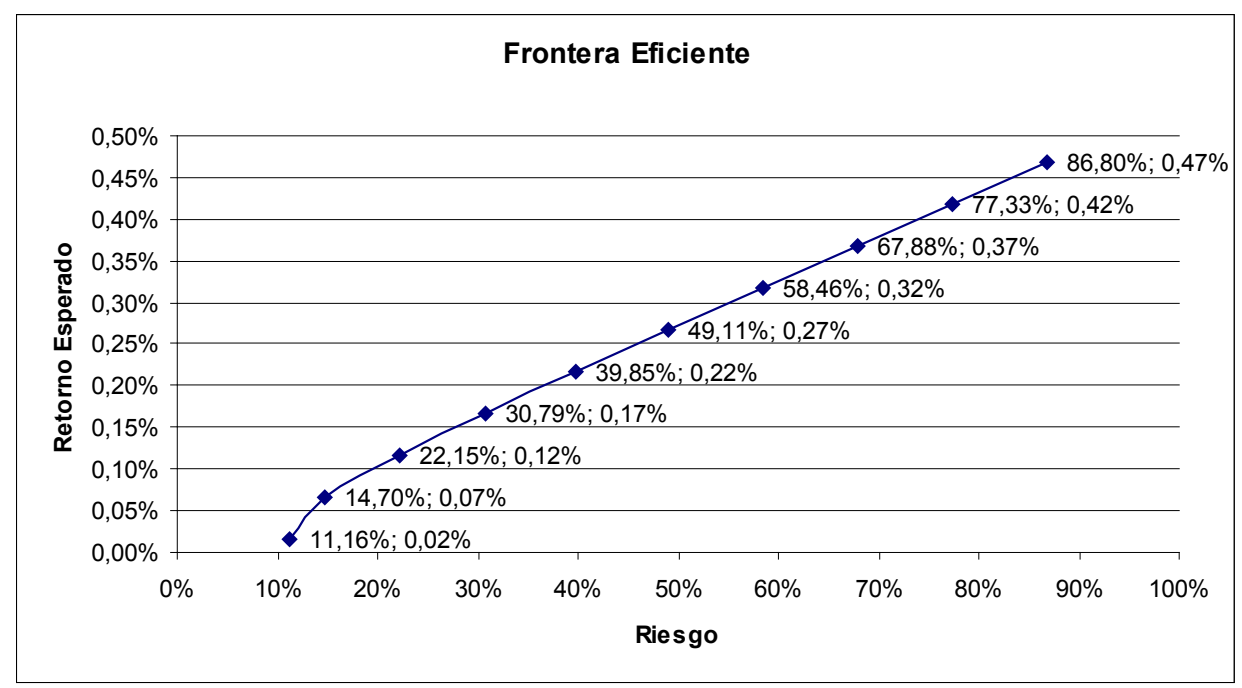

Se puede observar que a mayor rentabilidad o retorno esperado exigido, para las carteras, el riesgo (desviación estándar de la cartera) es cada vez mayor. Por tanto, para esperar obtener un retorno del $0.02 \%$ en la inversión, el riesgo que tendrá que asumir el inversionista será de un $11.16 \%$. Así se puede recorrer toda la curva del gráfico hasta llegar a la cartera que parece tener la mayor rentabilidad esperada de un $0.47 \%$ con un riesgo del $86,8 \%$.

En la tabla siguiente, se muestra el porcentaje que representan los montos a invertir en cada activo para las carteras que están sobre la frontera eficiente y que son los resultados determinados con el modelo.

Tabla 8: Rentabilidad y riesgo de cada cartera

\begin{tabular}{|l|c|c|}
\hline & Retorno & Desviación \\
\hline & Esperado cartera & Estándar cartera \\
\hline Cartera1 & $0,47 \%$ & $86,80 \%$ \\
\hline Cartera2 & $0,42 \%$ & $77,33 \%$ \\
\hline Cartera3 & $0,37 \%$ & $67,88 \%$ \\
\hline Cartera4 & $0,32 \%$ & $58,46 \%$ \\
\hline Cartera5 & $0,27 \%$ & $49,11 \%$ \\
\hline Cartera6 & $0,22 \%$ & $39,85 \%$ \\
\hline Cartera7 & $0,17 \%$ & $30,79 \%$ \\
\hline Cartera8 & $0,12 \%$ & $22,15 \%$ \\
\hline Cartera9 & $0,07 \%$ & $14,70 \%$ \\
\hline Cartera_min & $0,02 \%$ & $11,16 \%$ \\
\hline
\end{tabular}

La última fila de la tabla, corresponde a la cartera con el riesgo más pequeño que se puede tener al invertir en estos activos para obtener una cierta rentabilidad esperada. Esto quiere decir que rentabilidades que estén por debajo de dicho valor, tendrán un mayor nivel de riesgo, lo que desde el punto de vista de la teoría de cartera corresponderá a una inversión de carácter ineficiente, por lo tanto, el inversionista no tendrá más alternativa que moverse sobre la frontera eficiente.

También se puede observar, que a medida que va aumentando el nivel de rentabilidad esperada del portafolio, el modelo sugiere que el inversionista duplique y que algunas veces, casi hasta triplique los porcentajes del monto a invertir en los activos. Este hecho, observado hace suponer que los inversionistas deberán endeudarse para poder aspirar a obtener una rentabilidad cada vez mayor.

\section{Resultados modelo con estimación de matriz de varianza covarianza no condicional}

El modelo considera la misma información que el anterior, respecto de la media de los retornos para cada activo al 29 de diciembre de 2000, pero que es distinta para la matriz de varianza covarianza, información que se muestra en las Tabla 9 y Tabla 10. 
Tabla 9: Media de los retornos para cada activo en el período considerado

\begin{tabular}{|c|c|c|c|c|c|c|}
\hline Acción & COPEC & CTC-A & CAP & COLBUN & ENDESA & ENTEL \\
\hline Media & $0,021 \%$ & $0,040 \%$ & $-0,034 \%$ & $-0,028 \%$ & $-0,005 \%$ & $0,006 \%$ \\
\hline
\end{tabular}

Tabla 10: Matriz varianza covarianza no condicional (cifras en \%)

\begin{tabular}{|l|c|c|c|c|c|c|}
\hline & COPEC & CTC-A & CAP & COLBUN & ENDESA & ENTEL \\
\hline COPEC & $0,038 \%$ & $0,020 \%$ & $0,017 \%$ & $0,014 \%$ & $0,019 \%$ & $0,017 \%$ \\
\hline CTC-A & $0,020 \%$ & $0,043 \%$ & $0,015 \%$ & $0,013 \%$ & $0,021 \%$ & $0,014 \%$ \\
\hline CAP & $0,017 \%$ & $0,015 \%$ & $0,034 \%$ & $0,011 \%$ & $0,014 \%$ & $0,014 \%$ \\
\hline COLBUN & $0,014 \%$ & $0,013 \%$ & $0,011 \%$ & $0,044 \%$ & $0,014 \%$ & $0,011 \%$ \\
\hline ENDESA & $0,019 \%$ & $0,021 \%$ & $0,014 \%$ & $0,014 \%$ & $0,040 \%$ & $0,014 \%$ \\
\hline ENTEL & $0,017 \%$ & $0,014 \%$ & $0,014 \%$ & $0,011 \%$ & $0,014 \%$ & $0,046 \%$ \\
\hline
\end{tabular}

La construcción de la frontera eficiente, de acuerdo a las tablas 9 y 10, para obtener las carteras de inversión, se hace de la misma forma que el modelo anterior, lo cual entregó los siguientes resultados.

Las interpretaciones que se pueden hacer para esta frontera eficiente son las mismas que las de la anterior, pero en cuanto a los valores obtenidos para las rentabilidades de las carteras de inversión no se puede decir lo mismo. Respecto a esto, se puede observar que las rentabilidades que pueden esperar obtener el inversionista son bastante pequeñas, con valores para los riesgos también bastante pequeños. En el principio de la frontera eficiente, en el punto que corresponde al valor de mínimo global se puede observar que el inversionista obtiene rentabilidades esperadas negativas pero, al ir ascendiendo por la frontera eficiente las rentabilidades se hacen positivas; sin embargo, con valores bastante bajos.

Gráfico N. ${ }^{\circ}$ 2. Frontera eficiente matriz de covarianza no condicional

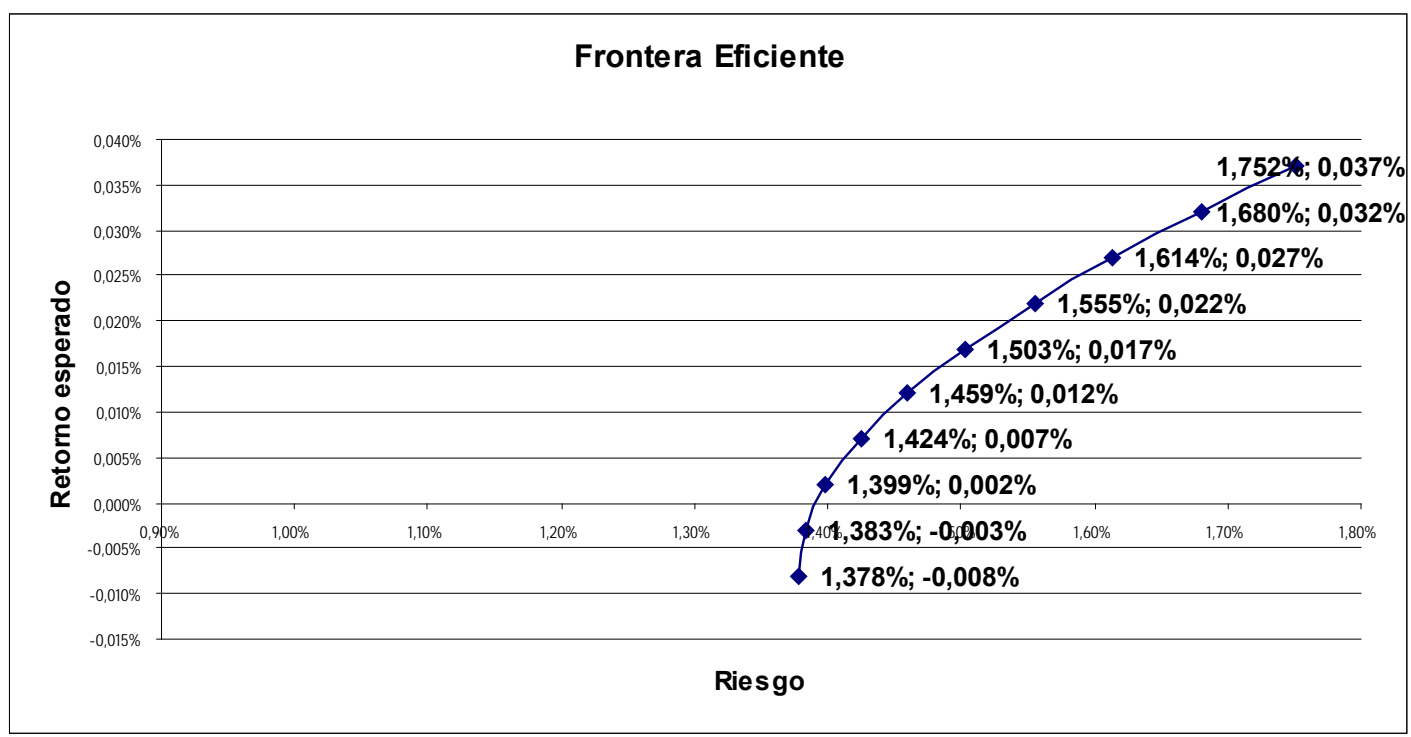

La siguiente tabla muestra las carteras que están sobre la frontera eficiente descrita y que muestran las ponderaciones de los montos a invertir en ellas. 
Tabla 11: Porcentajes invertidos por cada activo en la cartera con varianza covarianza no condicional.

\begin{tabular}{|l|c|c|c|c|c|c|c|c|}
\hline & & & \multicolumn{3}{|c|}{ MONTOS A INVERTIR (\%) } & & \\
\hline & Retorno & & & & & & Desviación \\
\hline & $\begin{array}{l}\text { Esperado } \\
\text { cartera }\end{array}$ & COPEC & CTC-A & CAP & COLBUN & ENDESA & ENTEL & $\begin{array}{c}\text { Estándar } \\
\text { cartera }\end{array}$ \\
\hline Cartera1 & $0,037 \%$ & $39,630 \%$ & $52,652 \%$ & $-18,096 \%$ & $0,312 \%$ & $2,081 \%$ & $23,420 \%$ & $1,752 \%$ \\
\hline Cartera2 & $0,032 \%$ & $36,324 \%$ & $48,042 \%$ & $-13,201 \%$ & $2,667 \%$ & $3,428 \%$ & $22,739 \%$ & $1,680 \%$ \\
\hline Cartera3 & $0,027 \%$ & $33,018 \%$ & $43,432 \%$ & $-8,306 \%$ & $5,022 \%$ & $4,776 \%$ & $22,058 \%$ & $1,614 \%$ \\
\hline Cartera4 & $0,022 \%$ & $29,712 \%$ & $38,822 \%$ & $-3,411 \%$ & $7,377 \%$ & $6,123 \%$ & $21,377 \%$ & $1,555 \%$ \\
\hline Cartera5 & $0,017 \%$ & $26,406 \%$ & $34,212 \%$ & $1,484 \%$ & $9,732 \%$ & $7,471 \%$ & $20,695 \%$ & $1,503 \%$ \\
\hline Cartera6 & $0,012 \%$ & $23,100 \%$ & $29,601 \%$ & $6,379 \%$ & $12,087 \%$ & $8,819 \%$ & $20,014 \%$ & $1,459 \%$ \\
\hline Cartera7 & $0,007 \%$ & $19,794 \%$ & $24,991 \%$ & $11,274 \%$ & $14,442 \%$ & $10,166 \%$ & $19,333 \%$ & $1,424 \%$ \\
\hline Cartera8 & $0,002 \%$ & $16,487 \%$ & $20,381 \%$ & $16,169 \%$ & $16,797 \%$ & $11,514 \%$ & $18,652 \%$ & $1,399 \%$ \\
\hline Cartera9 & $-0,003 \%$ & $13,181 \%$ & $15,771 \%$ & $21,064 \%$ & $19,152 \%$ & $12,861 \%$ & $17,971 \%$ & $1,383 \%$ \\
\hline Cartera_min & $-0,008 \%$ & $9,875 \%$ & $11,161 \%$ & $25,959 \%$ & $21,507 \%$ & $14,209 \%$ & $17,289 \%$ & $1,378 \%$ \\
\hline
\end{tabular}

Observando la tabla, se puede ver que las ponderaciones obtenidas en cada uno de los activos parecen ser más razonables que los del modelo anterior; sin embargo, la desventaja que presenta éste último es que sus retornos esperados son bastante pequeños.

En cuanto a los pesos, dentro de las carteras, los activos que más contribuyen son las acciones pertenecientes a: COPEC y CTC-A, teniendo menor ponderación las acciones correspondientes a: CAP, COLBUN y ENDESA.

\section{Medición del desempeño de las carteras}

La medición del desempeño de las carteras tiene como finalidad poder evaluar la gestión del portafolio basándonos en el riego-retorno de la cartera. Se han propuesto diversas alternativas entre las que se encuentra el índice de Sharpe, que es una medida que permite efectuar comparaciones entre distintos portafolios. Esta será la medida que se utiliza en este trabajo y que se define de la siguiente forma:

Donde:

$$
s_{p}=\frac{\bar{R}_{p}-R_{f}}{\sigma_{p}}
$$

$\bar{R}_{p}$ : es el retorno esperado del portafolio durante
el período de medición.
$R_{f}$ : es la tasa libre de riesgo

$\sigma_{p}$ : es la desviación estándar para el retorno del portafolio

Este índice mide la relación entre la prima de riesgo de la cartera $\bar{R}_{p}-R_{f}$ y su riesgo total $\sigma_{p}$.

Este índice fue calculado para el período que va desde el 3 de enero del 2000 al 29 de diciembre del mismo año, empleándose como tasa libre de riesgo los pagares reajustables del Banco Central (PRBC). Junto al cálculo del índice o (ratio Sharpe) se determina la rentabilidad del portafolio o cartera de mercado la cual corresponde al índice IGPA para el mismo período.

La utilización del IGPA como cartera de mercado o portafolio comparador (Benchmark) es que es un índice representativo del mercado chileno reflejando la globalidad de este en cuanto a rentabilidad $y$ riesgo.

Para determinar las variaciones del índice IGPA se empleó la siguiente relación:

Donde:

$$
R_{t}=\frac{I G P A_{t}}{I G P A_{t-1}}-1
$$

$R_{t}$ : es el retorno obtenido por el índice en el período considerado. 
$I G P A_{t}: \quad$ son los puntos obtenidos por el índice al período $\mathrm{t}$.

$I G P A_{t-1}$ : son los puntos obtenidos por el índice al período $\mathrm{t}-1$.
Las tablas muestran los desempeños obtenidos por las carteras determinadas con los modelos de media varianza, utilizando matrices de varianza covarianza condicionales y no condicionales.

Tabla12: Desempeño carteras para el período enero 2001 y febrero 2001.

\begin{tabular}{|l|c|c|c|c|c|c|}
\hline & \multicolumn{3}{|c|}{ NO CONDICIONAL } & \multicolumn{2}{c|}{ CONDICIONAL } & \\
\hline \multicolumn{1}{|c|}{ Fecha } & Retorno & Desviación & Ratio & Retorno & Desviación & Ratio \\
\hline Enero & Promedio & Estándar & Sharpe & Promedio & Estándar & Sharpe \\
\hline IGPA & $0,24 \%$ & $0,93 \%$ & $-5,48$ & $0,24 \%$ & $0,93 \%$ & $-5,48$ \\
\hline Cartera1 & $0,38 \%$ & $2,62 \%$ & $-1,89$ & $1,17 \%$ & $22,29 \%$ & $-0,19$ \\
\hline Cartera2 & $0,38 \%$ & $2,45 \%$ & $-2,02$ & $1,08 \%$ & $20,02 \%$ & $-0,21$ \\
\hline Cartera3 & $0,38 \%$ & $2,30 \%$ & $-2,15$ & $0,98 \%$ & $17,75 \%$ & $-0,24$ \\
\hline Cartera4 & $0,38 \%$ & $2,15 \%$ & $-2,3$ & $0,89 \%$ & $15,48 \%$ & $-0,29$ \\
\hline Cartera5 & $0,38 \%$ & $2,01 \%$ & $-2,46$ & $0,80 \%$ & $13,22 \%$ & $-0,34$ \\
\hline Cartera6 & $0,38 \%$ & $1,88 \%$ & $-2,62$ & $0,71 \%$ & $10,96 \%$ & $-0,42$ \\
\hline Cartera7 & $0,38 \%$ & $1,77 \%$ & $-2,79$ & $0,62 \%$ & $8,71 \%$ & $-0,54$ \\
\hline Cartera8 & $0,39 \%$ & $1,67 \%$ & $-2,95$ & $0,52 \%$ & $6,48 \%$ & $-0,74$ \\
\hline Cartera9 & $0,39 \%$ & $1,60 \%$ & $-3,1$ & $0,43 \%$ & $4,29 \%$ & $-1,14$ \\
\hline Cartera_min & $0,39 \%$ & $1,54 \%$ & $-3,21$ & $0,34 \%$ & $2,29 \%$ & $-2,18$ \\
\hline \multicolumn{1}{|c|}{ Fecha } & Retorno $\%$ & Desviación & Ratio $\%$ & Retorno & Desviación & Ratio \\
\hline Febrero & Promedio & Estándar & Sharpe & Promedio & Estándar & Sharpe \\
\hline IGPA & $-0,21 \%$ & $0,96 \%$ & $-6,09$ & $-0,21 \%$ & $0,96 \%$ & $-6,09$ \\
\hline Cartera1 & $-0,12 \%$ & $1,66 \%$ & $-3,47$ & $-0,45 \%$ & $9,28 \%$ & $-0,66$ \\
\hline Cartera2 & $-0,11 \%$ & $1,62 \%$ & $-3,55$ & $-0,41 \%$ & $8,34 \%$ & $-0,73$ \\
\hline Cartera3 & $-0,10 \%$ & $1,59 \%$ & $-3,62$ & $-0,37 \%$ & $7,41 \%$ & $-0,81$ \\
\hline Cartera4 & $-0,09 \%$ & $1,56 \%$ & $-3,67$ & $-0,32 \%$ & $6,48 \%$ & $-0,92$ \\
\hline Cartera5 & $-0,08 \%$ & $1,54 \%$ & $-3,72$ & $-0,28 \%$ & $5,55 \%$ & $-1,07$ \\
\hline Cartera6 & $-0,07 \%$ & $1,52 \%$ & $-3,75$ & $-0,24 \%$ & $4,65 \%$ & $-1,27$ \\
\hline Cartera7 & $-0,06 \%$ & $1,51 \%$ & $-3,77$ & $-0,20 \%$ & $3,77 \%$ & $-1,55$ \\
\hline Cartera8 & $-0,05 \%$ & $1,51 \%$ & $-3,78$ & $-0,16 \%$ & $2,93 \%$ & $-1,98$ \\
\hline Cartera9 & $-0,03 \%$ & $1,51 \%$ & $-3,77$ & $-0,12 \%$ & $2,19 \%$ & $-2,63$ \\
\hline Cartera_min & $-0,02 \%$ & $1,52 \%$ & $-3,74$ & $-0,08 \%$ & $1,70 \%$ & $-3,37$ \\
\hline & & & & & & \\
\hline
\end{tabular}

Para el mes de enero del 2001 se observa que todas las carteras presentan mayor rentabilidad que la cartera de mercado. Destacando las que fueron construidas con una estimación condicional de la matriz de varianza covarianza con rentabilidades notoriamente mayores que las del mercado representado por el IGPA Schwert (1989). Por otro lado, el ratio de Sharpe es negativo para todas las carteras, pero a pesar de ello, presenta un valor superior al de la cartera de mercado.

En el mes de febrero del 2001, se tienen carteras que con pérdidas menores que el mercado para las construidas con varianza covarianza no condicio- nal. En el caso de las carteras condicionales, la situación es distinta ya que estas pierden mucho más que el mercado.

\section{CONCLUSIONES}

Los modelos aplicados para la formación de carteras de inversión proporcionan cierto grado de cobertura frente al riesgo, evitando pérdidas mayores que las que tiene el mercado. A pesar de que los valores para los índices de desempeño utilizados son siempre negativos, los valores para las carteras de inversión en general son menores que los mostrados por el mercado. 
Otro aspecto interesante es que los modelos de cartera que emplearon en su formulación, las matrices de varianza covarianza condicionales, exhibieron la mayoría de las veces, retornos promedio superiores a los retornos promedio de carteras, sin usar varianza covarianza condicional para su formulación, lo que tal vez, sea indicio de que el empleo de los modelos GARCH en la estimación de la volatilidad condicional, de alguna manera logra capturar la información del mercado traspasándola al modelo de media varianza, lo que se traduce en que las ponderaciones para cada activo dentro de la cartera sean distintas a cuando se usa varianza covarianza no condicional.

Otro aspecto interesante en los modelos de cartera elaborados en base a varianza covarianza condicional es que presentan un mayor riesgo que los otros modelos, exhibiendo carteras con mayor variabilidad (mayor desviación estándar), lo que se traduce en mayores rentabilidades.

\section{REFERENCIAS BIBLIOGRÁFICAS}

[1] Carol, A. (2001). "Market Models: A guide to financial data analysis", ED. John Wiley, Nueva York.

[2] Engle R., Bollerslev T (1986). "Modelling the persistence of conditional variances". Econometric Reviews, Vol. 5, Issue 1, pp. 1-5.
[3] Fama E. (1963). Mandelbrot and the stable paretian Hypothesis. The journal of business pp. 420-445.

[4] Fernándes, C. (2006). Modelos GARCH para series financieras: Una aplicación al cálculo del valor en riesgo (VAR) - IAPUC-Insttituto de Gestao de Riscos Financeiros e Atuariais Departamento de Engenharia Eléctrica Brasil.

[5] Glosten L., Jagannathan R., Runkle D. (1993). On the relation between the expected value and the volatility of the nominal excess return on stocks. The journal of finance volumen $48 \mathrm{~N}^{\circ} 5$, pp. 1779-1795.

[6] Johnson C. (2000). "Métodos de evaluación del riesgo para portafolios de inversión". Banco central de Chile: documentos de trabajo, marzo, Vol. 67, pp. 7-13.

[7] Markowicz, H. (1959). "Portfolio selection: Efficient diversification of investment", ed John Wiley, Nueva York.

[8] Nelson D. (1991). Conditional heteroskedasticity in assets returns. A new approach econometrica. Vol. 54, № 2, pp. 347-370.

[9] Schwert W. (1989). "Why does stock market volatility change over time?" The journal of finance volumen $44, \mathrm{~N} .^{\circ} 5$, pp. 1115-1135.

[10] Sharpe W. (1970). "Portfolio Theory and Capital Market". Ed. McGraw-Hill. Nueva York. 